\title{
Systematic Review of Migraine Prophylaxis Adherence and Persistence
}

\author{
Zsolt Hepp, PharmD; Lisa M. Bloudek, PharmD, MS; and Sepideh F. Varon, PhD
}

\begin{abstract}
BACKGROUND: Migraine is a common neurological disease affecting $12 \%$ of Americans and millions worldwide. Medication adherence has been studied extensively in many chronic conditions, with poor adherence adversely affecting treatment outcomes. However, little is known about adherence to oral prophylaxis for migraine.
\end{abstract}

OBJECTIVE: To examine the literature on assessing oral prophylaxis medication adherence and persistence among migraine patients.

METHODS: A systematic search of the PubMed (1966 to present) and EMBASE (1974 to present) databases was conducted to locate prospective and retrospective observational studies and randomized controlled trials (RCTs) of propranolol, amitriptyline, and topiramate. RCTs were pooled, weighted by sample size, and stratified by drug and length of study. Average persistence rates and reasons for discontinuation cited in RCTs were examined for each medication.

RESULTS: A total of 788 unique articles were identified using the search criteria, 33 of which were included in the final review. Observational studies $(n=14)$ showed adherence ranges of $41 \%$ to $95 \%$ at 2 months, $21 \%$ to $80 \%$ at 6 months, and $35 \%$ to $56 \%$ at 12 months and persistence ranges of $41 \%$ to $88 \%$ at 2 months, $19 \%$ to $79 \%$ at 6 months, and $7 \%$ to $55 \%$ at 12 months. Pooled persistence from RCTs on propranolol, amitriptyline, and topiramate $(n=19)$ showed rates of $77 \%, 55 \%$, and $57 \%$, respectively, at 16-26 weeks. Adverse events were the most common reason for discontinuation cited ( $24 \%$ for topiramate and $17 \%$ for amitriptyline).

CONCLUSION: Observational studies and pooled data from RCTs demonstrate poor adherence and persistence to oral migraine prophylaxis.

J Manag Care Pharm. 2014;20(1):22-33

Copyright $\odot 2014$, Academy of Managed Care Pharmacy. All rights reserved.

\section{What is already known about this subject}

- Adherence measures provide a basic method for quantifying the extent to which patients follow a prescribed therapy over a specified time period. Generally, adherence is determined by calculating a ratio of doses taken on schedule compared with the total number of doses prescribed, while persistence is a measurement of the time during which a patient remains on a prescribed medication after initiating therapy.

- Chronic therapies are generally associated with poor adherence ( $<80 \%$ of medication taken as prescribed), which has been linked to poor outcomes. This lack of adherence has been demonstrated by large systematic literature reviews in various chronic conditions such as type 2 diabetes, hypertension, and osteoporosis. At the time of this review, no systematic literature reviews have been published on adherence or persistence to migraine prophylactics.

\section{What this study adds}

- This comprehensive review of the literature showed that migraine prophylactics are associated with poor adherence and persistence. This is the first such literature review for this class of medication

- This review is the first to include both observational studies and RCTs and to incorporate various methods for presenting adherence and persistence measures. It provides a summary of a wide range of data available on adherence and persistence related to migraine prophylaxis.

$\mathrm{M}$ igraine is a neurological condition that affects approximately $12 \%$ of patients in the United States and millions worldwide. ${ }^{1}$ Women are affected disproportionally, with a 3-fold higher prevalence of migraine disorder Prevalence peaks between age 25 and 55 years, affecting the most productive years of a person's life. ${ }^{2}$ Patients experience varying levels of frequency, severity, and disability associated with migraine. More frequent and severe cases with significant impact on patients' quality of life and daily activities may benefit from headache prophylaxis. Existing prophylaxis therapies include a variety of options (e.g., beta blockers, antidepressants, anticonvulsants, onabotulinumtoxinA). At the time of this study, guidelines, such as those published by the U.S. Headache Consortium, recommended first-line prophylaxis with propranolol, timolol, amitriptyline, or divalproex. ${ }^{3}$ Although some patients see relief from these therapies, unfavorable side effects may limit tolerability and thus impact the effectiveness of prophylaxis. Ultimately, patient tolerance is a crucial component for the success of migraine prophylaxis. A study that explored the extent to which migraine patients are willing to tolerate side effects of prophylaxis found that the top reasons why a prophylactic is deemed intolerable to patients are weight gain, memory loss, depression, and somnolence. ${ }^{4}$ These findings can be readily applied to migraine prophylaxis, where possible side effects include cognitive effects (topiramate), somnolence (amitriptyline and propranolol), and weight gain (divalproex).

Adherence refers to the extent to which a patient follows prescribed directions with respect to timing, dose, and frequency. ${ }^{5}$ Adherence to chronic therapy is a common issue across many disease states. Poor adherence $(<80 \%$ of medication taken as prescribed) has been associated with poor outcomes in a number of medication classes. ${ }^{6-10}$ 


\section{FIGURE 1 Results of Systematic Review (PubMed 1966-Present and EMBASE 1974-Present)}

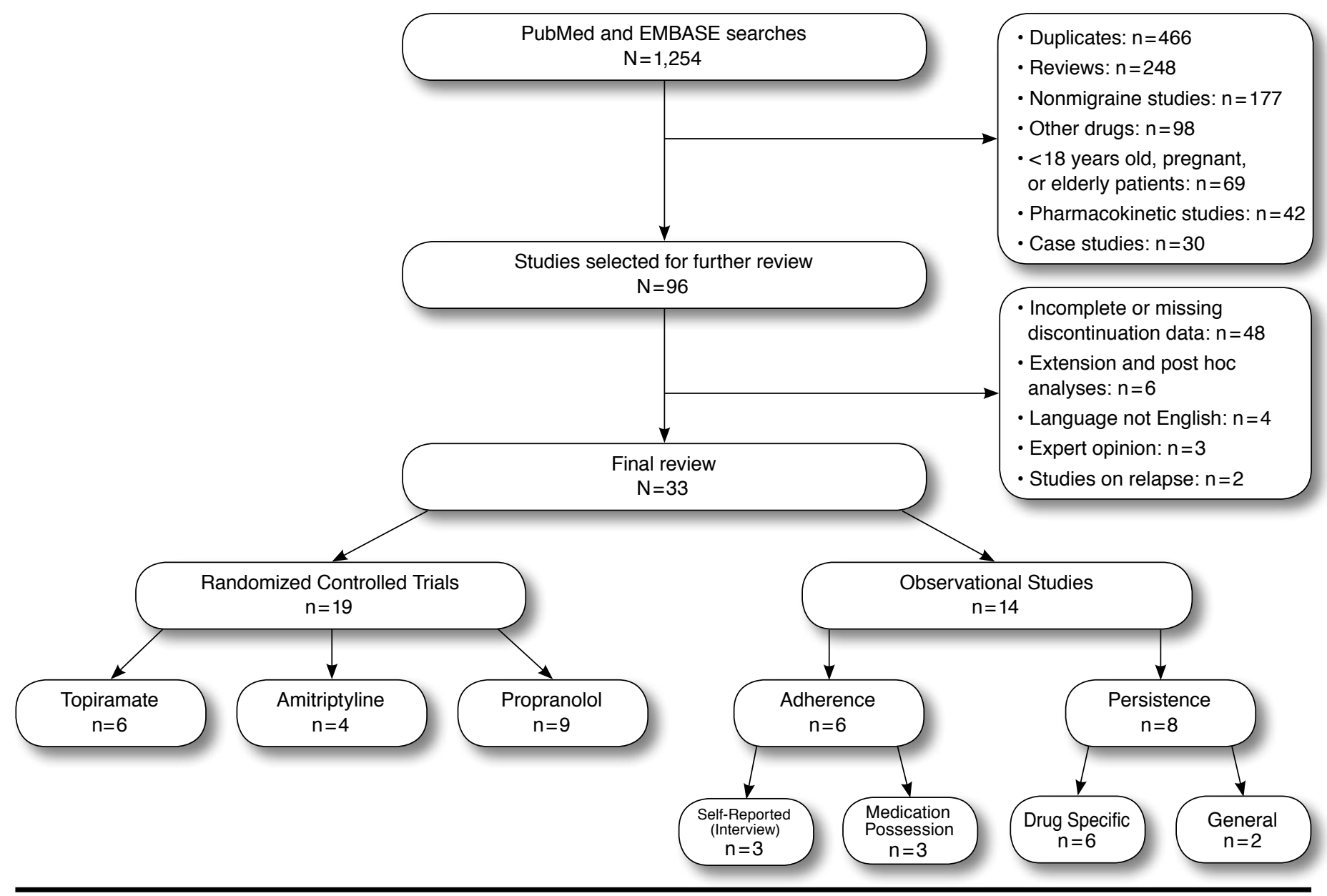

Medication adherence is often determined by a ratio of doses taken on schedule compared with the total number of doses prescribed over a specified period. ${ }^{11}$ In observational studies, this ratio can be determined through pill counts conducted by an investigator or logged by a medication event monitoring system (MEMS). Pill counts provide an accurate measure of adherence by comparing the prescribed quantity of drug with the actual quantity of drug taken by the patient over a period of time. MEMS has the additional benefit of providing an electronic time stamp that measures whether the drug is taken at the prescribed dosage interval. ${ }^{12}$ Another way to determine adherence is by calculating the medication possession ratio (MPR) or proportion of days covered (PDC) using pharmacy claims data. MPR is the sum of the days supply for all fill periods divided by the total number of days in that period. PDC is calculated as the number of days with drug on hand divided by the number of days in the specified time interval. ${ }^{13}$ MEMS is considered perhaps the most precise measure of adherence because it captures both quantity and dosage interval; however, claims database analyses and patient-reported adherence remain the most common methodologies employed due to the ability of conducting large studies quickly and at relatively low cost. ${ }^{14,15}$

Persistence refers to the time during which a patient remains on a prescribed medication after initiating therapy. While persistence can be measured directly by patient questionnaires and claims analyses, it may also be measured indirectly from discontinuation rates reported in observational and randomized controlled trials (RCTs). ${ }^{11}$ While RCTs employ a structured program of care that may result in low discontinuation rates compared with the real-world patient experience, trends of discontinuation over time, as well as reasons for discontinuation reported in RCTs, may yield important information on persistence.

Many comprehensive literature reviews have been conducted on the adherence and persistence to chronic 


\section{TABLE 1 Observational Studies on Oral Migraine Prophylaxis Adherence and Persistence}

\begin{tabular}{|c|c|c|c|c|c|c|c|c|c|c|}
\hline Reference & $\mathrm{N}$ & Design & $\begin{array}{l}\text { Study } \\
\text { Country }\end{array}$ & $\begin{array}{l}\text { Outcome } \\
\text { Measure }\end{array}$ & $\begin{array}{c}\text { Time } \\
\text { Horizon } \\
\text { (Weeks) }\end{array}$ & Treatment & $\begin{array}{c}\text { Mean } \\
\text { Age } \\
\end{array}$ & $\begin{array}{c}\% \\
\text { Female } \\
\end{array}$ & $\begin{array}{c}\text { Headache } \\
\text { Frequency } \\
\text { (Days/Month) }\end{array}$ & Results \\
\hline $\begin{array}{l}\text { Gaul } \\
2011^{16}\end{array}$ & 295 & $\begin{array}{c}\text { PRO, } \\
\text { PI }\end{array}$ & Germany & $\begin{array}{l}\text { Adherence } \\
\text { (self-reported: } \\
\text { headache diary } \\
\text { and phone } \\
\text { interview) }\end{array}$ & \begin{tabular}{|l|}
80 \\
\end{tabular} & $\begin{array}{l}\text { Topiramate, beta } \\
\text { blockers, magne- } \\
\text { sium, amitriptyline, } \\
\text { flunarizine, tiza- } \\
\text { nidine, opipramol, } \\
\text { duloxetine, valpro- } \\
\text { ate, butterbur, and } \\
\text { lamotrigine (any } \\
\text { dose of each) }\end{array}$ & 41 & 88.8 & $\begin{array}{l}\text { Tension head- } \\
\text { ache } 27(4-30) ; \\
\text { migraine } 11 \\
\text { (1-30); tension } \\
\text { + migraine } 18 \\
(2-30)\end{array}$ & $\begin{array}{l}91 \% \text { adherent at } \\
\text { first fill, } 39 \% \text { at } 5 \\
\text { months, and } 35 \% \text { at } \\
12-18 \text { months }\end{array}$ \\
\hline $\begin{array}{l}\text { Heckman } \\
2011^{17}\end{array}$ & 109 & $\begin{array}{l}\text { PRO, } \\
\text { PI }\end{array}$ & USA & $\begin{array}{l}\text { Adherence } \\
\text { (self-reported: } \\
\text { headache } \\
\text { diary) }\end{array}$ & 4 & $\begin{array}{l}\text { Antidepressants, } \\
\text { anticonvulsants, } \\
\text { beta blockers, } \\
\text { calcium blockers, } \\
\text { and other (non- } \\
\text { steroidal, low-dose } \\
\text { aspirin, Botox) (any } \\
\text { dose of each) }\end{array}$ & 39 & 90.5 & $17 \pm 7.7$ & $\begin{array}{l}78 \% \text { adherent at } \\
1 \text { month (reported } \\
\text { separately as } \\
69 \% \text { for African- } \\
\text { American patients } \\
\text { and } 82 \% \text { for } \\
\text { Caucasian patients) }\end{array}$ \\
\hline $\begin{array}{l}\text { Berger } \\
200920\end{array}$ & 5,187 & $\begin{array}{l}\text { RET, } \\
\text { CD }\end{array}$ & USA & $\begin{array}{l}\text { Adherence } \\
\text { (claims-based } \\
\text { MPR) }\end{array}$ & 24 & $\begin{array}{l}\text { All tricyclics and } \\
\text { SSRI antidepres- } \\
\text { sants, bupropion, } \\
\text { mirtazapine, trazo- } \\
\text { done, venlafaxine, } \\
\text { carbamazepine, } \\
\text { divalproex, gaba- } \\
\text { pentin, topiramate, } \\
\text { atenolol, meto- } \\
\text { prolol, nadolol, } \\
\text { propranolol, and } \\
\text { timolol (any dose of } \\
\text { each) }\end{array}$ & N/A & N/A & N/A & $\begin{array}{l}92.6 \% \text { adherent at } \\
1 \text { month, } 40.7 \% \text { at } \\
2 \text { months, } 30.9 \% \text { at } \\
3 \text { months, } 24.8 \% \text { at } \\
4 \text { months, } 24.3 \% \text { at } \\
5 \text { months, } 20.8 \% \text { at } \\
6 \text { months }\end{array}$ \\
\hline $\begin{array}{l}\text { Lafata } \\
2010^{19}\end{array}$ & 2,517 & $\begin{array}{l}\text { RET, } \\
\text { CD }\end{array}$ & USA & $\begin{array}{l}\text { Adherence } \\
\text { (claims-based } \\
\text { MPR) }\end{array}$ & 56 & $\begin{array}{l}\text { Amitriptyline, ven- } \\
\text { lafaxine, fluoxetine, } \\
\text { carbamazepine, gab- } \\
\text { apentin, topiramate, } \\
\text { prinivil, lisinopril, } \\
\text { candesartan, nife- } \\
\text { dipine, diltiazem, } \\
\text { verapamil, metopro- } \\
\text { lol, atenolol, inderal, } \\
\text { propranolol, and } \\
\text { timolol (any dose of } \\
\text { each) }\end{array}$ & 40 & 77 & N/A & $\begin{array}{l}56 \% \text { adherent at } 12 \\
\text { months }\end{array}$ \\
\hline $\begin{array}{l}\text { Rothrock } \\
2006^{18}\end{array}$ & 41 & $\begin{array}{l}\text { PRO, } \\
\text { SURV }\end{array}$ & USA & $\begin{array}{l}\text { Adherence } \\
\text { (self-reported: } \\
\text { headache diary) }\end{array}$ & 24 & $\begin{array}{l}\text { Anticonvulsants: } \\
\text { divalproex, topira- } \\
\text { mate, zonisamide, } \\
\text { or gabapentin (any } \\
\text { dose of each) }\end{array}$ & 42.5 & 92 & Mean 18 (SD N/A) & $\begin{array}{l}\text { Received patient edu- } \\
\text { cation, } 96 \% \text { adherent } \\
\text { at } 6 \text { months; no edu- } \\
\text { cation, } 58.5 \% \text { adher- } \\
\text { ence at } 6 \text { months; } \\
\text { composite } 79 \% \text { at } 6 \\
\text { months }\end{array}$ \\
\hline $\begin{array}{l}\text { Mulleners } \\
1998^{12}\end{array}$ & $\begin{array}{c}38 \\
\text { (11 lost } \\
\text { to } \\
\text { follow- } \\
\text { up) }\end{array}$ & $\begin{array}{l}\text { PRO, } \\
\text { SURV }\end{array}$ & $\begin{array}{l}\text { United } \\
\text { Kingdom }\end{array}$ & $\begin{array}{l}\text { Adherence } \\
\text { (pill count and } \\
\text { MEMS) }\end{array}$ & 8 & $\begin{array}{l}\text { Propranolol } 160 \mathrm{mg} \\
\text { qd, atenolol } 50 \mathrm{mg} \\
\text { bid, pizotifen } 0.5 \mathrm{mg} \text {, } \\
\text { or methysergide } \\
1 \mathrm{mg} \text { tid }\end{array}$ & N/A & 75.9 & $\begin{array}{l}\text { Mean or median } \\
\text { N/A, range } 2-8\end{array}$ & $\begin{array}{l}66.1 \% \text { adherent at } \\
2 \text { months }(79.8 \% \\
\text { for once daily, } 60 \% \\
\text { for twice daily, and } \\
54.2 \% \text { for } 3 \text { times } \\
\text { daily dosing) }\end{array}$ \\
\hline $\begin{array}{l}\text { Yaldo } \\
2008^{28}\end{array}$ & 12,783 & $\begin{array}{l}\text { RET, } \\
\text { CD }\end{array}$ & USA & $\begin{array}{l}\text { Persistence } \\
\text { (Kaplan-Meier } \\
\text { analysis of } \\
\text { claims database) }\end{array}$ & $\begin{array}{c}\text { N/A } \\
\text { (until } \\
\text { discon- } \\
\text { tinua- } \\
\text { tion) }\end{array}$ & $\begin{array}{l}\text { Amitriptyline, pro- } \\
\text { pranolol/timolol, } \\
\text { divalproex sodium, } \\
\text { and topiramate (any } \\
\text { dose of each) }\end{array}$ & $\begin{array}{l}42- \\
43.9\end{array}$ & 83.9 & N/A & $\begin{array}{l}92.4 \% \text { persistent at } \\
1 \text { month, } 41.0 \% \text { at } \\
2 \text { months, } 31.4 \% \text { at } \\
3 \text { months, } 25.2 \% \text { at } \\
4 \text { months, } 21.3 \% \text { at } \\
5 \text { months, } 18.5 \% \text { at } \\
6 \text { months, } 11.5 \% \text { at } \\
9 \text { months, } 7.3 \% \text { at } \\
12 \text { months }\end{array}$ \\
\hline
\end{tabular}




\section{TABLE 1 Observational Studies on Oral Migraine Prophylaxis Adherence and Persistence (continued)}

\begin{tabular}{|c|c|c|c|c|c|c|c|c|c|c|}
\hline Reference & $\mathrm{N}$ & Design & $\begin{array}{l}\text { Study } \\
\text { Country }\end{array}$ & $\begin{array}{l}\text { Outcome } \\
\text { Measure }\end{array}$ & $\begin{array}{c}\text { Time } \\
\text { Horizon } \\
\text { (Weeks) }\end{array}$ & Treatment & $\begin{array}{c}\text { Mean } \\
\text { Age }\end{array}$ & $\begin{array}{c}\% \\
\text { Female }\end{array}$ & $\begin{array}{l}\text { Headache } \\
\text { Frequency } \\
\text { (Days/Month) }\end{array}$ & Results \\
\hline $\begin{array}{l}\text { Krymchantowski } \\
2011^{25}\end{array}$ & 120 & $\begin{array}{l}\mathrm{MC} \\
\mathrm{PRO} \\
\mathrm{COM} \\
\mathrm{OL}\end{array}$ & Brazil & $\begin{array}{l}\text { Discontinuation } \\
\text { (headache diary) }\end{array}$ & \begin{tabular}{|l|}
12 \\
\end{tabular} & $\begin{array}{l}\text { Divalproex sodium } \\
500 \mathrm{mg} \text { bid vs. topi- } \\
\text { ramate } 75 \mathrm{mg} \text { bid }\end{array}$ & 41.2 & 86.6 & $8 \pm 4$ & $\begin{array}{l}65.8 \% \text { persistent at } \\
3 \text { months }\end{array}$ \\
\hline $\begin{array}{l}\text { Nelles } \\
2010^{21}\end{array}$ & 332 & $\begin{array}{l}\text { PRO, } \\
\text { MC, } \\
\text { OL, } \\
\text { NIS }\end{array}$ & Germany & $\begin{array}{l}\text { Discontinuation } \\
\text { (reported as part } \\
\text { of primary } \\
\text { outcome for } \\
\text { tolerability) }\end{array}$ & 56 & $\begin{array}{l}\text { Topiramate } \\
25-200 \mathrm{mg}\end{array}$ & 41.8 & 76.9 & N/A & $\begin{array}{l}77.7 \% \text { persistent at } \\
6 \text { months, } 60.4 \% \text { at } \\
9 \text { months, } 54.5 \% \text { at } \\
12 \text { months }\end{array}$ \\
\hline $\begin{array}{l}\text { Malessa } \\
2010^{22}\end{array}$ & 364 & $\begin{array}{l}\text { PRO, } \\
\text { MC, } \\
\text { OL, } \\
\text { SA }\end{array}$ & Germany & $\begin{array}{l}\text { Discontinuation } \\
\text { (secondary out- } \\
\text { come measure in } \\
\text { open-label study) }\end{array}$ & 24 & $\begin{array}{l}\text { Topiramate } \\
50-200 \mathrm{mg}\end{array}$ & 43.7 & 87.8 & $\begin{array}{l}\text { Mean } 8.84 \\
\text { (SD 3.28); } \\
\text { median } 8 \\
\text { (range 1-24) } \\
\end{array}$ & $\begin{array}{l}62.4 \% \text { persistent at } \\
6 \text { months, } 41.3 \% \text { at } \\
12 \text { months }\end{array}$ \\
\hline $\begin{array}{l}\text { Lampl } \\
200924\end{array}$ & 150 & $\begin{array}{l}\text { PRO, } \\
\text { MC, } \\
\text { OL }\end{array}$ & Austria & $\begin{array}{l}\text { Discontinuation } \\
\text { (secondary out- } \\
\text { come measure } \\
\text { in open-label } \\
\text { study) }\end{array}$ & 24 & $\begin{array}{l}\text { Amitriptyline ER } \\
25-50 \mathrm{mg} \text { qd }\end{array}$ & 32 & 73 & $\begin{array}{l}\text { Median } 7 \\
\text { (range 4-14) }\end{array}$ & $\begin{array}{l}88.0 \% \text { persistent } \\
\text { at end of } 2 \text { months } \\
\text { titration period, } \\
81.3 \% \text { at } 1 \text { month } \\
\text { (total } 3 \text { months), } \\
76.0 \% \text { at } 3 \text { months } \\
\text { (total } 6 \text { months) }\end{array}$ \\
\hline $\begin{array}{l}\text { Mei } \\
200423\end{array}$ & 185 & $\begin{array}{l}\text { PRO, } \\
\text { MC, } \\
\text { OL }\end{array}$ & Germany & $\begin{array}{l}\text { Discontinuation } \\
\text { (secondary out- } \\
\text { come measure in } \\
\text { open-label study) }\end{array}$ & 24 & $\begin{array}{l}\text { Topiramate } \\
100-200 \mathrm{mg} \text { qd }\end{array}$ & 45.7 & 90.7 & $\begin{array}{l}\text { Mean 6.2 } \\
\text { (SD 3.9); median } \\
6 \text { (range 0-23) }\end{array}$ & $\begin{array}{l}75 \% \text { persistent at } \\
1 \text { month, } 65 \% \text { at } \\
3 \text { months, } 59 \% \text { at } \\
6 \text { months }\end{array}$ \\
\hline $\begin{array}{l}\text { Rahimtoola } \\
200327\end{array}$ & 729 & $\begin{array}{l}\text { RET, } \\
\text { CD }\end{array}$ & Netherlands & $\begin{array}{l}\text { Discontinuation } \\
\text { (Kaplan-Meier } \\
\text { analysis of } \\
\text { claims database) }\end{array}$ & N/A & $\begin{array}{l}\text { Propranolol, meto- } \\
\text { prolol, pizotifen, } \\
\text { methysergide, flu- } \\
\text { narizine, clonidine, } \\
\text { valproic acid (any } \\
\text { dose of each) }\end{array}$ & 40 & 80.8 & N/A & $\begin{array}{l}48 \% \text { persistent at } \\
3 \text { months, } 34 \% \text { at } \\
6 \text { months, } 23 \% \text { at } \\
12 \text { months }\end{array}$ \\
\hline $\begin{array}{l}\text { Verspeelt } \\
1996^{26}\end{array}$ & 1,601 & $\begin{array}{l}\text { PRO, } \\
\text { MC, } \\
\text { COM }\end{array}$ & Belgium & $\begin{array}{l}\text { Discontinuation } \\
\text { (doctor surveys) }\end{array}$ & 32 & $\begin{array}{l}\text { Flunarizine } 5-10 \\
\text { mg qd, propranolol } \\
40-320 \mathrm{mg} \text { qd }\end{array}$ & 41 & 76.5 & N/A & $\begin{array}{l}65.3 \% \text { persistent at } \\
1 \text { month, } 50.6 \% \text { at } 4 \\
\text { months, } 40.6 \% \text { at } 8 \\
\text { months }\end{array}$ \\
\hline
\end{tabular}

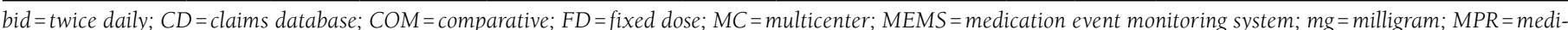

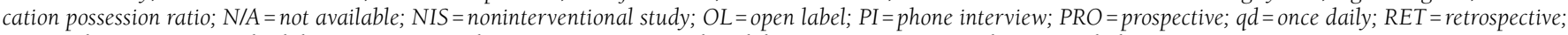
$S A=$ single arm; SD = standard deviation; SSRI= selective serotonin re-uptake inhibitor; SURV=survey; tid=3 times daily.

medications; however, none have been published on migraine prophylaxis to date. The purpose of this systematic literature review is to summarize all available evidence of adherence and persistence to 3 common oral medications used as migraine prophylaxis.

\section{Methods}

\section{Search Methods}

Potential studies were identified by searching the PubMed (1966 to July 1, 2011) and EMBASE (1974 to July 1, 2011) databases for "migraine prophylaxis" combined with the terms "adherence" OR "compliance" and "migraine prophylaxis" combined with each of the following medications: "propranolol" OR "amitriptyline" OR "topiramate" (Figure 1). The medications chosen for this review are the 3 most commonly used oral medications for migraine prophylaxis, and each is a member of a major therapeutic class of oral agents used as migraine prophylaxis (beta blockers, antidepressants, and anticonvulsants). Studies were limited to prospective and retrospective observational studies on adherence and persistence of migraine prophylaxis and RCTs of the 3 aforementioned migraine prophylaxis medications. Studies examining use for headache disorders other than migraine were excluded, as were editorials/commentaries, reviews, case studies, animal studies, cost-effectiveness studies, pharmacokinetic studies, and guidelines. Articles not available in English and studies conducted in children/adolescents ( $<18$ years of age), elderly, and pregnant subpopulations were also omitted.

\section{Data Synthesis}

Observational studies and RCTs were analyzed separately. Adherence and persistence data from observational studies were 


\section{TABLE 2 Persistence Data from Randomized Controlled Trials of Oral Migraine Prophylaxis}

\begin{tabular}{|c|c|c|c|c|c|c|c|c|c|c|c|}
\hline Reference & $\mathrm{N}$ & Design & $\begin{array}{l}\text { Study } \\
\text { Country }\end{array}$ & Inclusion Criteria & Exclusion Criteria & $\begin{array}{c}\text { Study } \\
\text { Length } \\
\text { (Weeks) }\end{array}$ & Treatment & $\begin{array}{l}\text { Baseline } \\
\text { Headache } \\
\text { Frequency } \\
\text { Mean (SD) }\end{array}$ & $\begin{array}{l}\text { Age } \\
\text { Mean } \\
\text { (SD) or } \\
\text { Median } \\
\text { (Range) }\end{array}$ & $\begin{array}{c}\% \\
\% \\
\text { Female }\end{array}$ & $\begin{array}{c}\text { Discon- } \\
\text { tinuation } \\
\text { Rates } \\
(\%)\end{array}$ \\
\hline \multirow[t]{2}{*}{$\begin{array}{l}\text { Mohammadianinejad } \\
2011^{31}\end{array}$} & \multirow[t]{2}{*}{80} & \multirow[t]{2}{*}{$\begin{array}{l}\text { DB, } \\
\text { PC, } \\
\text { RCT }\end{array}$} & \multirow[t]{2}{*}{ Iran } & \multirow{2}{*}{\begin{tabular}{|l|} 
Dx of migraine defined \\
by IHS for 1 yr, $4-15$ \\
migraines per month, \\
failed 1 or more pro- \\
phylactics, 1 yr after \\
menopause if menopause \\
already occurred, negative \\
pregnancy test and appro- \\
priate contraceptive use \\
during the study
\end{tabular}} & \multirow{2}{*}{$\begin{array}{l}\text { Dx of any other HA or } \\
\text { pain, pregnancy, prior use } \\
\text { of study medication }\end{array}$} & \multirow[t]{2}{*}{12} & $\begin{array}{l}\text { Topiramate } \\
100 \mathrm{mg} \text { qd }\end{array}$ & $\begin{array}{c}3.7 \\
(1.89)\end{array}$ & $\begin{array}{l}35.5 \\
(9.5)\end{array}$ & 77.5 & 5 \\
\hline & & & & & & & $\begin{array}{l}\text { Zonisamide } \\
200 \mathrm{mg} \mathrm{qd}\end{array}$ & $\begin{array}{l}3.6 \\
(1.42)\end{array}$ & $\begin{array}{c}33 \\
(9.2)\end{array}$ & 80 & 7.5 \\
\hline \multirow[t]{2}{*}{$\begin{array}{l}\text { Cady } \\
2011^{32}\end{array}$} & \multirow[t]{2}{*}{59} & \multirow[t]{2}{*}{$\begin{array}{l}\mathrm{MC} \\
\mathrm{DB} \\
\mathrm{RCT}\end{array}$} & \multirow[t]{2}{*}{ USA } & \multirow{2}{*}{$\begin{array}{l}\text { 18-65 yrs w/Dx of CM } \\
\text { defined by IHS } 2 \text { nd ed., } \\
\text { 3-8 migraine attacks per } \\
\text { month with avg. } 21 \mathrm{HA} \\
\text { days, negative pregnancy } \\
\text { test }\end{array}$} & \multirow{2}{*}{$\begin{array}{l}\text { Pregnant or breastfeeding, } \\
\text { Dx w/other HA besides } \\
\text { chronic migraine, renal or } \\
\text { liver impairment, previous } \\
\text { use of study medication, } \\
\text { ketogenic diet, alcohol/ } \\
\text { drug abuse, or overuse of } \\
\text { acute medications }\end{array}$} & \multirow[t]{2}{*}{12} & $\begin{array}{l}\text { Topiramate } \\
100-200 \mathrm{mg} \text { qd }\end{array}$ & 20.5 & \multirow[t]{2}{*}{$\begin{array}{c}39.6 \\
(19.6- \\
64)\end{array}$} & \multirow[t]{2}{*}{91.5} & 26.7 \\
\hline & & & & & & & $\begin{array}{l}\text { OnabotulinumtoxinA } \\
100-200 \text { units } \\
\text { injection }\end{array}$ & 21.8 & & & 24.1 \\
\hline \multirow[t]{2}{*}{$\begin{array}{l}\text { Couch } \\
2010^{33}\end{array}$} & \multirow[t]{2}{*}{391} & \multirow[t]{2}{*}{$\begin{array}{l}\text { MC, } \\
\text { DB, } \\
\text { PC, } \\
\text { RCT }\end{array}$} & \multirow[t]{2}{*}{ USA } & \multirow[t]{2}{*}{$\begin{array}{l}\text { 18-70 yrs w/Dx of } \\
\text { migraine as defined by } \\
\text { the } 1962 \text { Ad Hoc National } \\
\text { Institutes of Health } \\
\text { Committee and the } 1988 \\
\text { ICHD Guidelines with at } \\
\text { least } 2 \text { moderate or worse } \\
\text { migraines per month }\end{array}$} & \multirow{2}{*}{$\begin{array}{l}\text { Secondary HA, pregnant } \\
\text { or nursing mothers, uri- } \\
\text { nary retention, glaucoma, } \\
\text { cardiac disease, sustained } \\
\text { hypertension, concurrent } \\
\text { guanethidine or MAOIs, } \\
\text { prostate hypertrophy, } \\
\text { thyroid disease, concur- } \\
\text { rent thyroid medications, } \\
\text { seizure disorders, current } \\
\text { anti-migraine agent or } \\
\text { known hypersensitivity to } \\
\text { study medication }\end{array}$} & \multirow[t]{2}{*}{20} & $\begin{array}{l}\text { Amitriptyline } \\
25-100 \mathrm{mg} \text { qd }\end{array}$ & \multirow[t]{2}{*}{ N/A } & 34.1 & 79 & 48 \\
\hline & & & & & & & Placebo qd & & 34.0 & 83 & 54 \\
\hline \multirow[t]{2}{*}{$\begin{array}{l}\text { Rodriguez-Leyva } \\
2010^{34}\end{array}$} & \multirow[t]{2}{*}{36} & \multirow[t]{2}{*}{$\begin{array}{c}\mathrm{DB} \\
\mathrm{COM} \\
\mathrm{RCT}\end{array}$} & \multirow[t]{2}{*}{ Mexico } & \multirow{2}{*}{$\begin{array}{l}\text { 18-60 yrs w/Dx of } \\
\text { migraine defined by IHS } \\
\text { at least } 6 \text { months prior } \\
\text { to study start date and } \\
\text { before age } 40 \text { with } 3 \text { or } \\
\text { more migraine events per } \\
\text { month }\end{array}$} & \multirow{2}{*}{$\begin{array}{l}\text { Pregnancy or lactation, } \\
\text { Dx of other HA, history of } \\
\text { renal lithiasis, glaucoma, } \\
\text { schizophrenia, bipolar } \\
\text { disorder, infectious, } \\
\text { immunologic, cardio- } \\
\text { vascular, prostatic, or } \\
\text { metabolic disease, known } \\
\text { hypersensitivity to study } \\
\text { medication }\end{array}$} & \multirow[t]{2}{*}{16} & $\begin{array}{l}\text { Amitriptyline 25-50 } \\
\text { mg qd }\end{array}$ & N/A & $\begin{array}{l}31.6 \\
(9.6)\end{array}$ & 88.9 & 27.8 \\
\hline & & & & & & & $\begin{array}{l}\text { Topiramate 25-50 } \\
\text { mg qd }\end{array}$ & N/A & $\begin{array}{c}36.3 \\
(10.5)\end{array}$ & 83.3 & 22.2 \\
\hline \multirow[t]{3}{*}{$\begin{array}{l}\text { Domingues } \\
2009^{35}\end{array}$} & \multirow[t]{3}{*}{76} & \multirow{3}{*}{$\begin{array}{l}\mathrm{DB} \\
\mathrm{COM} \\
\mathrm{RCT}\end{array}$} & \multirow[t]{3}{*}{ Brazil } & \multirow{3}{*}{$\begin{array}{l}>18 \text { yrs w/Dx of migraine } \\
\text { or chronic migraine } \\
\text { defined by IHS } 2 \text { nd ed. } \\
\text { with } 4 \text { or more attacks of } \\
\text { migraine per month. No } \\
\text { prior or current use of a } \\
\text { prophylactic was allowed }\end{array}$} & $\begin{array}{l}\text { Bronchial asthma, symp- } \\
\text { tomatic hypotension, }\end{array}$ & 12 & $\begin{array}{l}\text { Propranolol } \\
40 \mathrm{mg} \text { bid } \\
\end{array}$ & N/A & N/A & N/A & 44 \\
\hline & & & & & $\begin{array}{l}\text { hyperlactemia, bladder } \\
\text { retention, known hyper- }\end{array}$ & & $\begin{array}{l}\text { Nortriptyline } \\
20 \mathrm{mg} \text { bid } \\
\end{array}$ & N/A & N/A & N/A & 42 \\
\hline & & & & & cation & & $\begin{array}{l}\text { Propranolol } 40 \mathrm{mg}+ \\
\text { nortriptyline } \\
20 \mathrm{mg} \text { bid }\end{array}$ & N/A & N/A & N/A & 41 \\
\hline $\begin{array}{l}\text { Dodick } \\
2009^{36}\end{array}$ & 331 & $\begin{array}{l}\mathrm{MC}, \\
\mathrm{DB} \\
\mathrm{COM} \\
(\mathrm{NI}) \\
\mathrm{RCT}\end{array}$ & USA & $\begin{array}{l}\geq 18 \text { yrs w/Dx of migraine } \\
\text { defined by IHS for at } \\
\text { least } 6 \text { months and } 3-12 \\
\text { (but not more than } 15 \text { ) } \\
\text { migraines per month for } 3 \\
\text { months prior to study date }\end{array}$ & $\begin{array}{l}\text { Previously failed }>2 \text { trials } \\
\text { of migraine preventative } \\
\text { medication (trial lasting } \\
\geq 3 \text { months) or had failed } \\
\text { trial of topiramate or } \\
\text { amitriptyline because of a } \\
\text { lack of efficacy or adverse } \\
\text { event, using abortive } \\
\text { HA medication for }>15 \\
\text { days per month, onset }\end{array}$ & 26 & $\begin{array}{l}\text { Topiramate } \\
50 \mathrm{mg} \text { bid }\end{array}$ & $\begin{array}{l}6.1 \\
(2.7)\end{array}$ & $\begin{array}{c}39.7 \\
(10.7)\end{array}$ & 86.6 & 40.7 \\
\hline & & & & & $\begin{array}{l}\text { of migraine after age } 50 \text {, } \\
\text { other type HA, contrain- } \\
\text { dication to study medica- } \\
\text { tions, unstable medical } \\
\text { condition in the last } 2 \text { yrs, } \\
\text { psychiatric disorder in } \\
\text { the last } 6 \text { months, drug } \\
\text { or alcohol abuse, liver } \\
\text { disease, pregnant or nurs- } \\
\text { ing, not practicing birth } \\
\text { control }\end{array}$ & & $\begin{array}{l}\text { Amitriptyline } \\
100 \mathrm{mg} \text { qd }\end{array}$ & $\begin{array}{c}6.2 \\
(2.7)\end{array}$ & $\begin{array}{c}37.9 \\
(11.3)\end{array}$ & 83 & 40.3 \\
\hline
\end{tabular}




\section{TABLE 2 Persistence Data from Randomized Controlled Trials of Oral Migraine Prophylaxis (continued)}

\begin{tabular}{|c|c|c|c|c|c|c|c|c|c|c|c|}
\hline Reference & $\mathrm{N}$ & Design & $\begin{array}{l}\text { Study } \\
\text { Country }\end{array}$ & Inclusion Criteria & Exclusion Criteria & $\begin{array}{c}\text { Study } \\
\text { Length } \\
\text { (Weeks) }\end{array}$ & Treatment & $\begin{array}{l}\text { Baseline } \\
\text { Headache } \\
\text { Frequency } \\
\text { Mean (SD) }\end{array}$ & \begin{tabular}{|c|} 
Age \\
Mean \\
(SD) or \\
Median \\
(Range)
\end{tabular} & $\begin{array}{c} \\
\% \\
\text { Female } \\
\end{array}$ & \begin{tabular}{|c} 
Discon- \\
tinuation \\
Rates \\
$(\%)$
\end{tabular} \\
\hline \multirow[t]{4}{*}{$\begin{array}{l}\text { Adelman } \\
2008^{37}\end{array}$} & \multirow[t]{4}{*}{1,580} & \multirow{4}{*}{\begin{tabular}{|c|} 
PD \\
from \\
pivotal \\
DB, \\
PC, \\
RCT
\end{tabular}} & \multirow[t]{4}{*}{ USA } & \multirow{4}{*}{\begin{tabular}{|l|}
$12-65$ yrs w/Dx of \\
migraine with or without \\
aura defined by IHS at \\
least $6-12$ months prior \\
to study start date with \\
$3-12$ but no more than \\
15) migraine events per \\
month during l month \\
prior to study, postmeno- \\
pausal or practicing birth \\
control
\end{tabular}} & \multirow{4}{*}{\begin{tabular}{|l|} 
Dx for another type of \\
HA, migraine started after \\
age 50 , failed 2 or more \\
doses of migraine prophy- \\
lactic, overuse of anal- \\
gesics, triptans, or other \\
acute migraine treatments, \\
concomitant prophylactic, \\
diagnosed and treated \\
depression 3 months prior \\
to study start date, neph- \\
rolithiasis, participants \\
of previous topiramate or \\
other experimental study \\
30 days prior to screening \\
\end{tabular}} & \multirow[t]{4}{*}{$20-26$} & $\begin{array}{l}\text { Topiramate } \\
50 \mathrm{mg} \text { qd }\end{array}$ & N/A & \begin{tabular}{|c|}
39.5 \\
$(11.8)$
\end{tabular} & 87 & 46 \\
\hline & & & & & & & $\begin{array}{l}\text { Topiramate } \\
100 \mathrm{mg} \text { qd }\end{array}$ & N/A & $\begin{array}{l}39.9 \\
(11.4)\end{array}$ & 86 & 38 \\
\hline & & & & & & & $\begin{array}{l}\text { Topiramate } \\
200 \mathrm{mg} \text { qd }\end{array}$ & N/A & $\begin{array}{l}40.5 \\
(11.8)\end{array}$ & 85 & 46 \\
\hline & & & & & & & $\begin{array}{l}\text { Placebo } \\
\text { qd }\end{array}$ & N/A & $\begin{array}{l}40.1 \\
(10.9)\end{array}$ & 83 & 35 \\
\hline \multirow[t]{2}{*}{$\begin{array}{l}\text { Ashtari } \\
2008^{38}\end{array}$} & \multirow[t]{2}{*}{62} & \multirow[t]{2}{*}{$\begin{array}{c}\text { DB, } \\
\text { COM, } \\
\text { RCT }\end{array}$} & \multirow[t]{2}{*}{ Iran } & \multirow{2}{*}{\begin{tabular}{|l|}
$12-65$ yrs w/Dx of \\
migraine with or without \\
aura defined by IHS at \\
least 1 yr prior to study \\
start date with 3 or more \\
migraine events per \\
month during 3 months \\
prior to study, onset $<50$ \\
yrs of age, pain free inter- \\
val of 48 hours between \\
attacks, concomitant pro- \\
phylactics discontinued \\
1 month prior to entry \\
into trial \\
\end{tabular}} & \multirow[t]{2}{*}{\begin{tabular}{|l|} 
Pregnant or breast feed- \\
ing, concomitant medical \\
condition including renal \\
stones, cardiac, liver, or \\
neoplastic diseases, and \\
neurologic disorders
\end{tabular}} & \multirow[t]{2}{*}{8} & $\begin{array}{l}\text { Topiramate } \\
50 \mathrm{mg} \text { qd }\end{array}$ & $\begin{array}{l}6.07 \\
(1.89)\end{array}$ & $\begin{array}{l}29.9 \\
(9)\end{array}$ & 86.7 & 3.2 \\
\hline & & & & & & & $\begin{array}{l}\text { Propranolol } \\
80 \mathrm{mg} \text { qd }\end{array}$ & $\begin{array}{c}5.83 \\
(1.98)\end{array}$ & $\begin{array}{l}31.7 \\
(8)\end{array}$ & 76.7 & 3.2 \\
\hline \multirow[t]{3}{*}{$\begin{array}{l}\text { Keskinbora } \\
2008^{39}\end{array}$} & \multirow[t]{3}{*}{75} & \multirow{3}{*}{$\begin{array}{l}\text { DB, } \\
\text { COM, } \\
\text { RCT }\end{array}$} & \multirow[t]{3}{*}{ Turkey } & \multirow{3}{*}{$\begin{array}{l}\text { 18-60 yrs w/Dx of } \\
\text { migraine with or with- } \\
\text { out aura defined by IHS } \\
\text { within } 1 \text { year of study } \\
\text { start date, and migraine } \\
\text { frequency of } 3-12 \text { (max } \\
\text { 15) with pain intensity }>5 \\
\text { over } 3 \text { months preceding } \\
\text { study }\end{array}$} & \multirow{3}{*}{$\begin{array}{l}\text { Use of ergots or triptans } \\
\text { and/or another prophy- } \\
\text { lactic within } 4 \text { weeks of } \\
\text { study start day, severe } \\
\text { depression, other type } \\
\text { HA, allergy to topiramate } \\
\text { and/or amitriptyline, and } \\
\text { hepatic, renal, or heart } \\
\text { disease }\end{array}$} & \multirow[t]{3}{*}{12} & \begin{tabular}{|l} 
Topiramate \\
$25-200 \mathrm{mg}$ qd
\end{tabular} & $\begin{array}{c}6.3 \\
(3.25) \\
\end{array}$ & $\begin{array}{r}32.3 \\
(9.39) \\
\end{array}$ & 70 & 16.7 \\
\hline & & & & & & & $\begin{array}{l}\text { Amitriptyline } \\
\text { 10-150 mg qd }\end{array}$ & $\begin{array}{c}6.09 \\
(2.56) \\
\end{array}$ & $\begin{array}{c}37.9 \\
(8.67) \\
\end{array}$ & 63.6 & 21.4 \\
\hline & & & & & & & $\begin{array}{l}\text { Topiramate } \\
25-200+ \\
\text { amitriptyline } \\
10-150 \mathrm{mg} \text { qd }\end{array}$ & $\begin{array}{l}6.05 \\
(2.75)\end{array}$ & $\begin{array}{l}39.1 \\
(9.13)\end{array}$ & 66.7 & 8.7 \\
\hline \multirow[t]{2}{*}{$\begin{array}{l}\text { Krymchantowski } \\
2002^{40}\end{array}$} & \multirow[t]{2}{*}{39} & \multirow[t]{2}{*}{$\begin{array}{l}\mathrm{DB}, \\
\mathrm{COM}\end{array}$} & \multirow[t]{2}{*}{ Brazil } & \multirow{2}{*}{$\begin{array}{l}\text { Dx with transformed HA } \\
\text { from overusing symptom- } \\
\text { atic medications }\end{array}$} & \multirow{2}{*}{ N/A } & \multirow[t]{2}{*}{9} & $\begin{array}{l}\text { Amitriptyline } \\
20 \mathrm{mg} \text { bid } \\
\end{array}$ & 27.4 & \multirow[t]{2}{*}{$\begin{array}{l}36.4 \\
(2.5)\end{array}$} & 63.2 & 32 \\
\hline & & & & & & & $\begin{array}{l}\text { Amitriptyline } 20 \mathrm{mg} \\
\text { + fluoxetine } \\
20 \mathrm{mg} \text { bid }\end{array}$ & 24.2 & & 70 & 30 \\
\hline \multirow[t]{3}{*}{$\begin{array}{l}\text { Diener } \\
2002^{41}\end{array}$} & \multirow[t]{3}{*}{808} & \multirow[t]{3}{*}{$\begin{array}{c}\mathrm{MC} \\
\mathrm{DB} \\
\mathrm{COM} \\
(\mathrm{EQ})\end{array}$} & \multirow[t]{3}{*}{$\begin{array}{l}\text { Germany, } \\
\text { Spain, } \\
\text { Switzerland, } \\
\text { Italy, } \\
\text { Belgium }\end{array}$} & \multirow{3}{*}{\begin{tabular}{|l|}
$18-65$ yrs w/Dx of \\
migraine with or with- \\
out aura defined by IHS \\
within 1 yr of study date \\
and a migraine frequency \\
of 2 to 6 migraines per \\
month over the 2 months \\
preceding the study; inter- \\
val HA permitted only if \\
occurrence $<6 /$ month
\end{tabular}} & \begin{tabular}{|l|} 
Any prophylactic use 2 \\
months preceding study, \\
depression, extrapyra- \\
midal disorders, asthma, \\
COPD, bronchospasms, \\
heart failure, AV block,
\end{tabular} & 16 & $\begin{array}{l}\text { Flunarizine } \\
5 \mathrm{mg} \text { qd }\end{array}$ & $\begin{array}{c}3.1 \\
(2.3-3.9)\end{array}$ & $\begin{array}{c}37 \\
(18-66)\end{array}$ & 79.1 & 16.7 \\
\hline & & & & & $\begin{array}{l}\text { hypotension, peripheral } \\
\text { vascular disease, diabetes, } \\
\text { hepatic, renal cardiovas- } \\
\text { cular, malignant, or respi- } \\
\text { ratory disease, alcohol// } \\
\text { drug dependence, preg- }\end{array}$ & & $\begin{array}{l}\text { Flunarizine } \\
10 \mathrm{mg} \text { qd } \\
\text { ( } 5 \text { days of the week) }\end{array}$ & $\begin{array}{c}3.0 \\
(2.1-3.8)\end{array}$ & $\begin{array}{c}37 \\
(18-63)\end{array}$ & 81.8 & 19.3 \\
\hline & & & & & $\begin{array}{l}\text { nancy, lactation, child- } \\
\text { bearing potential without } \\
\text { birth control, no pain- } \\
\text { free interval of } 24 \text { hours } \\
\text { between migraine attacks, } \\
\text { poor compliance during } \\
\text { run-in period }\end{array}$ & & $\begin{array}{l}\text { Propranolol } \\
160 \mathrm{mg} \text { qd }\end{array}$ & $\begin{array}{c}3.0 \\
(2.9-3.2)\end{array}$ & $\begin{array}{c}37 \\
(17-65)\end{array}$ & 83.3 & 16.7 \\
\hline $\begin{array}{l}\text { Rao } \\
2000^{42}\end{array}$ & 259 & $\begin{array}{l}\text { DB, } \\
\text { COM }\end{array}$ & India & $\begin{array}{l}\text { Patients attending an } \\
\text { outpatient department of }\end{array}$ & N/A & 12 & $\begin{array}{l}\text { Cyproheptadine } \\
2 \mathrm{mg} \text { bid }\end{array}$ & N/A & $\begin{array}{c}28.6 \\
(16-53)\end{array}$ & 67.2 & 23.6 \\
\hline & & & & $\begin{array}{l}\text { neurology and neurosur- } \\
\text { gery and had } 2 \text { or more }\end{array}$ & & & $\begin{array}{l}\text { Propranolol } \\
40 \mathrm{mg} \text { bid } \\
\end{array}$ & & & & 17.7 \\
\hline & & & & & & & $\begin{array}{l}\text { Cyproheptadine } \\
2 \mathrm{mg}+\text { propranolol } \\
40 \mathrm{mg} \text { bid }\end{array}$ & & & & 11.6 \\
\hline & & & & & & & Placebo & & & & 30.4 \\
\hline
\end{tabular}




\section{TABLE 2 Persistence Data from Randomized Controlled Trials of Oral Migraine Prophylaxis (continued)}

\begin{tabular}{|c|c|c|c|c|c|c|c|c|c|c|c|}
\hline Reference & $\mathrm{N}$ & Design & $\begin{array}{l}\text { Study } \\
\text { Country }\end{array}$ & Inclusion Criteria & Exclusion Criteria & $\begin{array}{c}\text { Study } \\
\text { Length } \\
\text { (Weeks) }\end{array}$ & Treatment & $\begin{array}{l}\text { Baseline } \\
\text { Headache } \\
\text { Frequency } \\
\text { Mean (SD) }\end{array}$ & \begin{tabular}{|c|} 
Age \\
Mean \\
(SD) or \\
Median \\
(Range)
\end{tabular} & \begin{tabular}{|c|}
$\%$ \\
Female \\
\end{tabular} & $\begin{array}{l}\text { Discon- } \\
\text { tinuation } \\
\text { Rates } \\
(\%)\end{array}$ \\
\hline \multirow[t]{3}{*}{$\begin{array}{l}\text { Kaniecki } \\
1997^{43}\end{array}$} & \multirow[t]{3}{*}{37} & \multirow[t]{3}{*}{$\begin{array}{l}\mathrm{SB}, \mathrm{PC} \\
\mathrm{CO} \\
\mathrm{RCT}\end{array}$} & \multirow[t]{3}{*}{ USA } & \multirow{3}{*}{$\begin{array}{l}18-60 \text { yrs w/Dx of } \\
\text { migraine with or with- } \\
\text { out aura defined by IHS } \\
\text { within } 1 \text { year of study } \\
\text { start date, and migraine } \\
\text { frequency of } 3-12 \text { (max } \\
\text { 15) with pain intensity }>5 \\
\text { over } 3 \text { months preceding } \\
\text { study. }\end{array}$} & \multirow{3}{*}{$\begin{array}{l}\text { Past trial of divalproex } \\
\text { or propranolol, failure } \\
\text { of } 2 \text { trials of migraine } \\
\text { prophylactic, severe medi- } \\
\text { cal or psychiatric illness, } \\
\text { analgesic use }>15 \text { days } \\
\text { per month, alcohol/drug } \\
\text { abuse, lack of contracep- } \\
\text { tion use by potential } \\
\text { childbearing women }\end{array}$} & \multirow[t]{3}{*}{12} & $\begin{array}{l}\text { Propranolol } \\
60 \mathrm{mg} \text { tid }\end{array}$ & \multirow[t]{3}{*}{$\begin{array}{l}4.38 \\
(2-8)\end{array}$} & \multirow[t]{3}{*}{ N/A } & \multirow[t]{3}{*}{81.8} & 3 \\
\hline & & & & & & & $\begin{array}{l}\text { Divalproex } \\
500 \mathrm{mg} \text { tid }\end{array}$ & & & & 11 \\
\hline & & & & & & & Placebo & & & & 0 \\
\hline \multirow[t]{2}{*}{$\begin{array}{l}\text { Bank } \\
1994^{44}\end{array}$} & \multirow[t]{2}{*}{64} & \multirow{2}{*}{$\begin{array}{l}\text { DB, } \\
\text { COM, } \\
\text { RCT }\end{array}$} & \multirow[t]{2}{*}{ Hungary } & \multirow[t]{2}{*}{$\begin{array}{l}\text { Patients w/Dx of migraine } \\
\text { defined by IHS }\end{array}$} & \multirow[t]{2}{*}{ N/A } & \multirow[t]{2}{*}{12} & $\begin{array}{l}\text { Fluvoxamine } \\
50 \mathrm{mg} \text { qd }\end{array}$ & \multirow[t]{2}{*}{ N/A } & $\begin{array}{l}34.5 \\
(7.4)\end{array}$ & 71.9 & 15.6 \\
\hline & & & & & & & $\begin{array}{l}\text { Amitriptyline } \\
25 \mathrm{mg} \text { qd }\end{array}$ & & $\begin{array}{l}33.5 \\
(8.3)\end{array}$ & 75 & 31.3 \\
\hline \multirow[t]{2}{*}{$\begin{array}{l}\text { Pradalier } \\
1989^{45}\end{array}$} & \multirow[t]{2}{*}{74} & \multirow[t]{2}{*}{$\begin{array}{l}\text { MC, } \\
\text { DB, } \\
\text { PC, } \\
\text { RCT }\end{array}$} & \multirow[t]{2}{*}{ France } & \multirow[t]{2}{*}{$\begin{array}{l}\text { 18-65 yrs w/Dx of } \\
\text { migraine with or without } \\
\text { aura defined by IHS for at } \\
\text { least } 2 \text { yrs preceding the } \\
\text { study and had } 2-8 \text { crises } \\
\text { per month }\end{array}$} & \multirow{2}{*}{$\begin{array}{l}\text { No prior prophylactic } \\
\text { treatment during } 2 \text { weeks } \\
\text { preceding the study, con- } \\
\text { gestive heart failure, heart } \\
\text { block, bradycardia ( }<50 \\
\text { beats/min), Raynaud's, } \\
\text { high blood pressure, resis- } \\
\text { tance to } 2 \text { previous trials } \\
\text { of migraine prophylactics, } \\
\text { no other concomitant } \\
\text { medication (only usual } \\
\text { acute migraine medica- } \\
\text { tions allowed) }\end{array}$} & \multirow[t]{2}{*}{16} & $\begin{array}{l}\text { Propranolol LA } \\
160 \mathrm{mg} \text { qd }\end{array}$ & $\begin{array}{c}1.66 \\
(0.23) \\
\text { per week }\end{array}$ & $\begin{array}{l}37.1 \\
(1.7)\end{array}$ & 77.5 & 45.0 \\
\hline & & & & & & & Placebo & $\begin{array}{c}1.40 \\
(0.20) \\
\text { per week }\end{array}$ & $\begin{array}{l}37.7 \\
(1.8)\end{array}$ & 73.5 & 44.1 \\
\hline \multirow[t]{3}{*}{$\begin{array}{l}\text { Mikkelsen } \\
1986^{46}\end{array}$} & \multirow[t]{3}{*}{39} & \multirow{3}{*}{$\begin{array}{l}\mathrm{DB} \\
\mathrm{PC} \\
\mathrm{CO} \\
\mathrm{RCT}\end{array}$} & \multirow[t]{3}{*}{ Sweden } & \multirow{3}{*}{$\begin{array}{l}\text { 18-65 yrs w/Dx of com- } \\
\text { mon or classic migraine } \\
\text { defined by the } 1962 \\
\text { Ad Hoc Committee on } \\
\text { Classification of Headache } \\
\text { for at least } 1 \text { yr preceding } \\
\text { the study and at least } 3 \\
\text { attacks per month }\end{array}$} & \multirow{3}{*}{$\begin{array}{l}\text { Allergy to study medica- } \\
\text { tion; serious heart, kidney, } \\
\text { liver, or psychiatric condi- } \\
\text { tions; asthma, bronchitis, } \\
\text { diabetes, active ulceration, } \\
\text { pregnancy or breast feed- } \\
\text { ing; concomitant migraine } \\
\text { prophylactic within l } \\
\text { month of study start date }\end{array}$} & \multirow[t]{3}{*}{12} & $\begin{array}{l}\text { Propranolol } \\
40 \mathrm{mg} \text { tid }\end{array}$ & \multirow[t]{3}{*}{ N/A } & \multirow[t]{3}{*}{$\begin{array}{c}38 \\
(15-65)\end{array}$} & N/A & 7.7 \\
\hline & & & & & & & $\begin{array}{l}\text { Tolfenamic acid } \\
100 \mathrm{mg} \text { tid }\end{array}$ & & & & 7.7 \\
\hline & & & & & & & Placebo & & & & 5.1 \\
\hline $\begin{array}{l}\text { Ryan } \\
1984^{47}\end{array}$ & 48 & \begin{tabular}{|c|} 
DB, \\
COM, \\
RCT
\end{tabular} & USA & $\begin{array}{l}\text { 21-65 yrs w/primary } \\
\text { Dx of common or clas- } \\
\text { sic migraine, at least } 3 \\
\text { migraine attacks per } \\
\text { month }\end{array}$ & \begin{tabular}{|l|} 
Pregnancy or not taking \\
birth control if of poten- \\
tial childbearing age, \\
hypersensitivity to beta \\
blockers, significant GI
\end{tabular} & 12 & $\begin{array}{l}\text { Nadolol } \\
80 \mathrm{mg} \text { qd }\end{array}$ & 6.13 & N/A & 72.9 & 6.3 \\
\hline & & & & & $\begin{array}{l}\text { disorder, cluster HA, CHF, } \\
\text { sinus bradycardia, severe } \\
\text { mitral or aortic valve dis- } \\
\text { ease, COPD, CAD, asthma, } \\
\text { diabetes, recent MI, }\end{array}$ & & $\begin{array}{l}\text { Nadolol } \\
160 \mathrm{mg} \text { qd }\end{array}$ & 5.56 & & & 0 \\
\hline & & & & & $\begin{array}{l}\text { hepatic or renal impair- } \\
\text { ment, hyperthyroidism, } \\
\text { any psychosis or central } \\
\text { nervous system disease, } \\
\text { or intracranial disease }\end{array}$ & & \begin{tabular}{|l|} 
Propranolol \\
$160 \mathrm{mg}$ qd
\end{tabular} & 7.42 & & & 12.5 \\
\hline $\begin{array}{l}\text { Tfelt-Hansen } \\
1984^{48}\end{array}$ & 96 & $\begin{array}{l}\mathrm{MC} \\
\mathrm{DB}\end{array}$ & $\begin{array}{l}\text { Denmark, } \\
\text { Norway, }\end{array}$ & $\begin{array}{l}18-65 \text { yrs w/ } 2-6 \text { com- } \\
\text { mon migraine attacks per }\end{array}$ & $\begin{array}{l}\text { Any other type of HA, } \\
\text { head injury, contraindica- }\end{array}$ & 12 & $\begin{array}{l}\text { Timolol } \\
10 \mathrm{mg} \text { bid }\end{array}$ & $\begin{array}{c}5.97 \\
(3.39)\end{array}$ & $\begin{array}{l}39.5 \\
(9.9)\end{array}$ & N/A & 19.7 \\
\hline & & $\begin{array}{l}\mathrm{PC} \\
\mathrm{CO} \\
\mathrm{RCT}\end{array}$ & & $\begin{array}{l}\text { month as defined by the } \\
1962 \text { Ad Hoc Committee } \\
\text { on Classification of }\end{array}$ & $\begin{array}{l}\text { tions to beta blockers, } \\
\text { fertile women not using } \\
\text { birth control, women on }\end{array}$ & & $\begin{array}{l}\text { Propranolol } \\
80 \mathrm{mg} \text { bid }\end{array}$ & & & & 15.6 \\
\hline & & & & Headache & $\begin{array}{l}\text { oral contraceptives, heart } \\
\text { rate }<54 \text { beats/min and/ } \\
\text { or diastolic blood pressure } \\
\leq 100 \mathrm{mmHg}\end{array}$ & & Placebo & & & & 13.5 \\
\hline $\begin{array}{l}\text { Andersson } \\
1981^{49}\end{array}$ & 49 & $\begin{array}{c}\mathrm{DB} \\
\mathrm{COM} \\
\mathrm{CO}\end{array}$ & Denmark & $\begin{array}{l}\text { 22-68 yrs w/at least } 3 \\
\text { attacks per month in } \\
\text { the preceding } 2 \text { months } \\
\text { defined as paroxysmal } \\
\text { HA associated with dis- }\end{array}$ & \begin{tabular}{|l|} 
Previous treatment w/ \\
propranolol, contraindica- \\
tion to beta blocker, treat- \\
ment with neuroleptic \\
drugs, thymoleptic drugs,
\end{tabular} & 12 & $\begin{array}{l}\text { Propranolol } \\
80 \mathrm{mg} \text { bid }\end{array}$ & N/A & 38 & 69.4 & 10.2 \\
\hline & & & & $\begin{array}{l}\text { comfort, possibly inability } \\
\text { to work, and l or more } \\
\text { of the following: nausea, } \\
\text { vomiting, visual distur- } \\
\text { bances, and paresthesia }\end{array}$ & $\begin{array}{l}\text { or minor tranquilizers, } \\
\text { serious liver or kidney } \\
\text { diseases }\end{array}$ & & $\begin{array}{l}\text { Femoxetine } \\
200 \mathrm{mg} \text { bid }\end{array}$ & & & & 14.3 \\
\hline
\end{tabular}

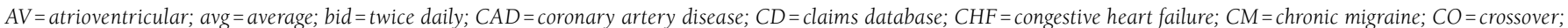
$C O M=$ comparative $; C O P D=$ chronic obstructive pulmonary disease; $D B=$ double blind; $D x=$ diagnosis; $E Q=$ equivalence; FD=fixed dose; $G I=$ gastrointestinal; HA = headache; $I C H D=$ International Classification of Headache Disorders; IHS = International Headache Society; MAOI= monoamine oxidase inhibitor; MC = multicenter; MEMS = medication

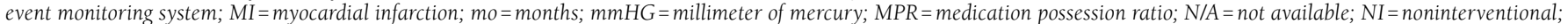

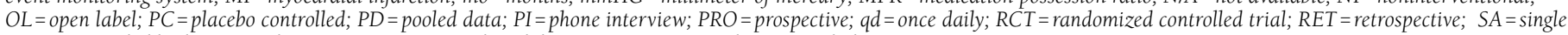
arm; SB =single blind; SSRI= selective serotonin re-uptake inhibitor; SUR=survey; tid=3 times daily; yrs=years. 


\begin{tabular}{|c|c|c|}
\hline & \multicolumn{2}{|c|}{ Study Length } \\
\hline & 8-12 Weeks & 16-26 Weeks \\
\hline \multicolumn{3}{|l|}{ Topiramate } \\
\hline Total number of patients & 125 & 1,331 \\
\hline Number of patients who discontinued & 15 & 573 \\
\hline Discontinuation rate & $12.0 \%$ & $43.1 \%$ \\
\hline Persistence (1-discontinuation rate) & $88.0 \%$ & $56.9 \%$ \\
\hline \multicolumn{3}{|l|}{ Amitriptyline } \\
\hline Total number of patients & 79 & 381 \\
\hline Number of patients who discontinued & 24 & 172 \\
\hline Discontinuation rate & $30.4 \%$ & $45.1 \%$ \\
\hline Persistence (1-discontinuation rate) & $69.6 \%$ & $54.9 \%$ \\
\hline \multicolumn{3}{|l|}{ Propranolol } \\
\hline Total number of patients & 355 & 453 \\
\hline Number of patients who discontinued & 49 & 104 \\
\hline Discontinuation rate & $13.8 \%$ & $23.0 \%$ \\
\hline Persistence (1-discontinuation rate) & $86.2 \%$ & $77.0 \%$ \\
\hline \multicolumn{3}{|l|}{ Placebo } \\
\hline Total number of patients & 241 & 676 \\
\hline Number of patients who discontinued & 36 & 275 \\
\hline Discontinuation rate & $14.9 \%$ & $40.7 \%$ \\
\hline Persistence (1-discontinuation rate) & $85.1 \%$ & $59.3 \%$ \\
\hline
\end{tabular}

summarized as part of the systematic review. Discontinuation data from RCTs were pooled, weighted by sample size, and stratified by drug and length of study. Average persistence rates (1-discontinuation) and reasons for discontinuation cited in RCTs were examined for each medication.

\section{Results}

\section{Search Results}

Searches returned a total of 1,254 articles, of which 788 were found to be unique. Abstracts were reviewed for 96 studies that met the inclusion/exclusion criteria, of which 48 were subsequently excluded because of incomplete or missing discontinuation data. The absence of data was, in part, caused by a number of crossover studies, especially among propranolol RCTs, that did not report discontinuation rates in the study results. A further 4 studies were available only in Spanish $(n=1)$, Portuguese $(n=1)$, or German $(n=2) ; 6$ reported data on patients included in other studies (open-label extension studies or post hoc analyses); 2 examined relapse after discontinuing the study medication; and 3 were expert opinion. Included studies $(\mathrm{N}=33)$ are shown in Table 1 , which lists observational studies that examined adherence and persistence, and Table 2 , which lists the RCTs of the 3 prophylactic medications and the rate of discontinuation reported in each study.

\section{Observational Studies: Adherence}

Of the 6 observational studies that examined migraine prophylaxis adherence, 1 used electronic MEMS to assess adherence, ${ }^{12}$ while 3 reported outcomes based on self-report, ${ }^{16-18}$ and 2 used retrospective claims analysis to calculate MPR. ${ }^{19,20}$ Five of the 6 studies were conducted in the United States, with time horizons ranging from 4 to 56 weeks. The sixth study was conducted in Germany and used a time horizon of 80 weeks. All studies used a wide variety of medications from the 3 major therapeutic classes of oral migraine prophylaxis agents-anticonvulsants, antidepressants, and antihypertensives-except for 1 , which restricted the study medications to anticonvulsants. ${ }^{18}$ Medication dosage was not considered in any of the studies. Across studies, adherence ranged from $41 \%$ to $95 \%$ at 2 months and declined over time to $21 \%$ to $80 \%$ at 6 months and $35 \%$ to $56 \%$ at 12 months. Individual study data are shown in Table 1.

\section{Observational Studies: Persistence}

Four open-label studies were identified that evaluated topiramate ${ }^{21-23}$ or amitriptyline alone, ${ }^{24}$ while 2 comparative studies evaluated divalproex and topiramate, ${ }^{25}$ and flunarizine and propranolol. ${ }^{26}$ Studies were conducted in Germany, ${ }^{21-23}$ Austria, ${ }^{24}$ Belgium, ${ }^{26}$ the Netherlands, ${ }^{27}$ and Brazil. ${ }^{25}$ Dosages varied by individual patient but were within the range of approved FDA-labeled dose for migraine prophylaxis, where applicable. Persistence was also evaluated in 2 large claims database analyses that included a wide range of medications from the 3 major classes of oral migraine prophylaxis, without regard for dose..$^{27,28}$ These studies report that persistence ranged from $41 \%$ to $88 \%$ at 2 months, $19 \%$ to $79 \%$ at 6 months, and $7 \%$ to $55 \%$ at 12 months. Individual study data are shown in Table 1.

\section{Randomized Controlled Trials: Persistence}

Since persistence can be calculated as the inverse of discontinuation, persistence can be inferred from discontinuation rates reported in RCTs. Nineteen RCTs provided sufficient data on discontinuation, as part of trial design, among propranolol, amitriptyline, and topiramate. The populations of migraine patients were generally similar, as evidenced by the similar age and gender distributions and comparable recruitment criteria (inclusion/exclusion) across the RCTs reviewed. Differences were assumed to be negligible; therefore, results were pooled and weighted by sample size to calculate a weighted average discontinuation rate for each medication of interest (Table 3). Although some studies evaluated medications other than those included in this review, only patients on propranolol, amitriptyline, topiramate, and placebo were pooled. Time horizons varied across studies, ranging from 8 weeks to 26 


\section{FIGURE 2 Combined Weighted Persistence Rates from Randomized Controlled Trials}

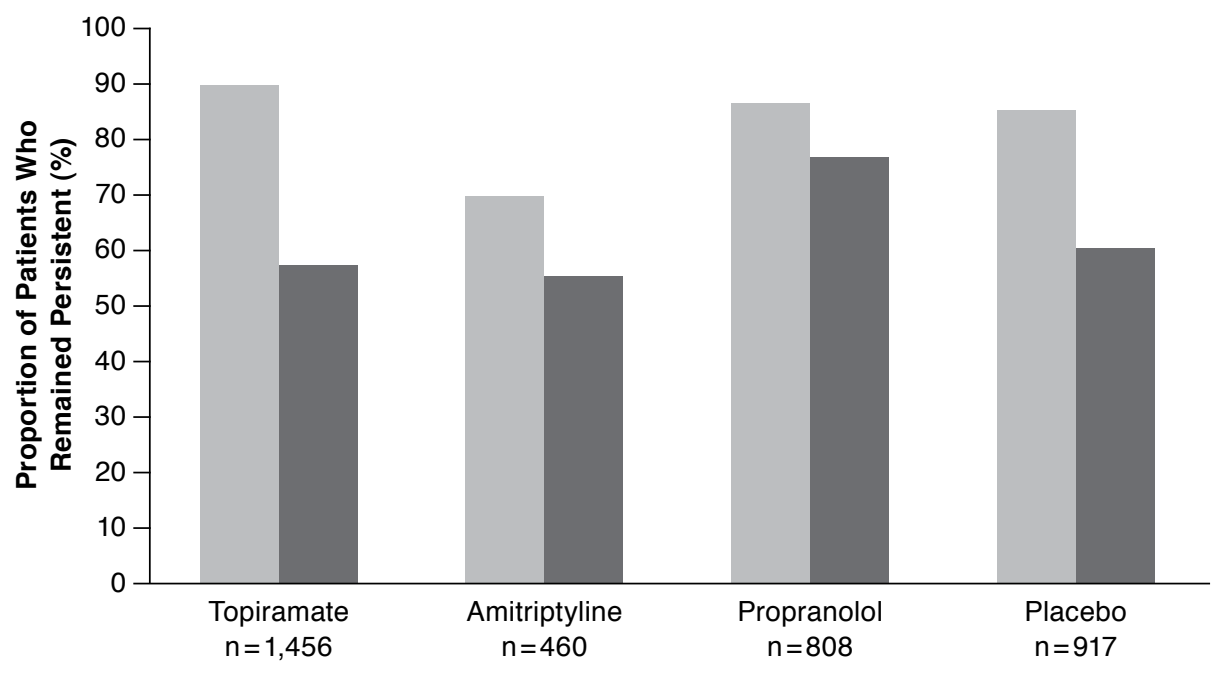

8-12 weeks

16-26 weeks weeks. This difference was adjusted for by stratifying into 2 separate groups: 8-12 weeks and 16-26 weeks. The weighted average discontinuation rate of each medication and placebo were compared for each time horizon group and presented in Figure 2 as the proportion of patients who stayed persistent (calculated as 1-discontinuation at the end of the study time horizon). The primary reasons for discontinuation across the different medications were also compared and summarized in Table 4 as well as Figure 3. The pooled data show that persistence declines rapidly over time, with nearly half of RCT participants discontinuing from clinical trials of amitriptyline and topiramate by $12-26$ weeks ( $45 \%$ and $43 \%$, respectively). Adverse events were the most common reason for discontinuation cited, especially among patients treated with topiramate (24\%) and amitriptyline (17\%). Individual study data from each RCT are available in Table 2.

\section{Discussion}

This review demonstrated a downward trend in migraine prophylaxis adherence and persistence over time that is similar to that seen for other chronic disease states. Adherence and persistence were shown to be below the acceptable threshold $(<80 \%)$ after 6 months among both observational and RCT data. While study heterogeneity limits the ability to compare across all available data, this review of the literature has produced several important findings. First, there was a substantially lower rate of discontinuation among RCTs evaluating propranolol compared with amitriptyline or topiramate. The reason for this low rate of discontinuation among patients taking propranolol is unknown. However, the propranolol RCTs

\begin{tabular}{l|c|c|c|c}
\hline TABLE 4 & \multicolumn{5}{|c}{ Discontinuation Rates by Cause } \\
\hline & $\begin{array}{c}\text { Topiramate } \\
(\%)\end{array}$ & $\begin{array}{c}\text { Amitriptyline } \\
(\%)\end{array}$ & $\begin{array}{c}\text { Propranolol } \\
(\%)\end{array}$ & $\begin{array}{c}\text { Placebo } \\
(\%)\end{array}$ \\
\hline Adverse events & 23.70 & 16.74 & 7.77 & 6.96 \\
\hline Patient choice & 5.56 & 18.56 & 1.21 & 6.25 \\
\hline Lost to follow-up & 3.43 & 6.30 & 2.95 & 3.77 \\
\hline Other & 8.45 & 9.53 & 6.97 & 15.45 \\
\hline
\end{tabular}

were generally conducted earlier (1980s) than the other 2 medications reviewed, which may have influenced RCT design, data capture, or documentation of study discontinuation. In addition, the types of RCTs for propranolol are largely small studies, potentially reducing the discontinuation rate if trial participants were more closely managed.

Aside from differences in study duration, RCTs were not adjusted for differences in study design, and this absence of adjustment may bias the results if there were systematic differences in the rate of discontinuation in amitriptyline, propranolol, and topiramate RCTs that are unrelated to the study drug. Counterintuitively, pooled discontinuation rates for the placebo groups across all studies had similar rates of discontinuation compared with amitriptyline and topiramate. However, when stratified by primary reported reason for discontinuation, placebo differs from active agents in the drivers of discontinuation. Placebo has very low rates of discontinuation because of adverse events and very high discontinuation for other reasons (including lack of therapeutic effect), while discontinuation of active interventions was mostly attributed to adverse events. 


\section{FIGURE 3 Weighted Discontinuation Rate by Cause}

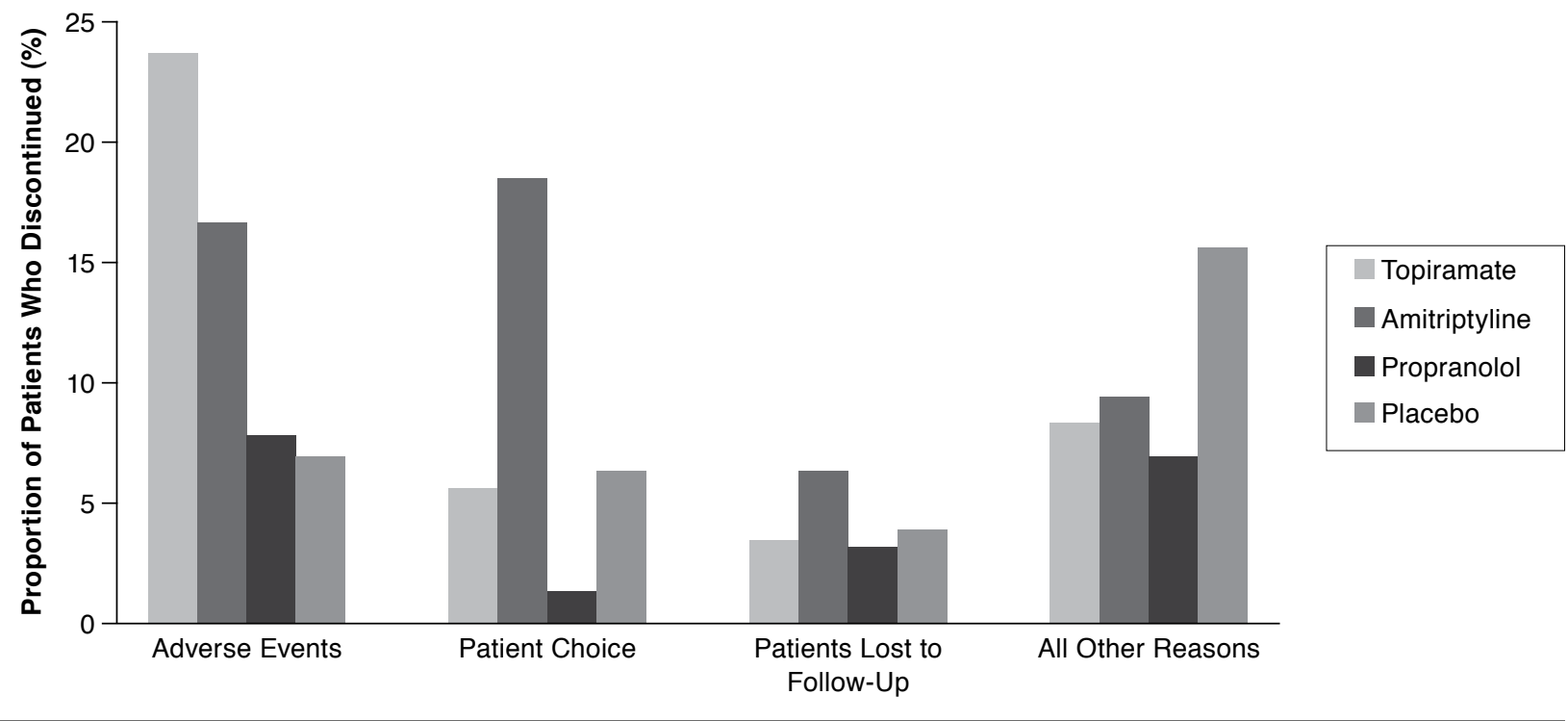

\section{Limitations}

This review is subject to several important limitations. There are many oral agents used for migraine prophylaxis. For this systematic review, only the 3 most common oral medications were reviewed. However, the 3 medications described here are each a member of the 3 major therapeutic classes of oral agents used as migraine prophylaxis (anticonvulsants, antidepressants, and beta blockers) and thus may be somewhat representative of the therapeutic class as a whole. Another important note is that pooling results across studies may not be appropriate, given differences in study design, geographic region population, dosing, etc. For this reason, we refrained from combining the results of observational studies. The discontinuation rates and reason for study withdrawal from RCTs may not be generalizable to the real-world patient experience of these agents because of the structured program of care employed in clinical trials. We acknowledge that such bias exists, but that it is likely to produce a conservative estimate of persistence rates, as those enrolled in a clinical trial are potentially less likely to discontinue taking the drug. Furthermore, in 3 of the 14 observational studies, adherence was calculated using patient-reported data. While this methodology allows for convenient real-world data collection, it has been shown to overestimate adherence rates. ${ }^{29,30}$ Conversely, using MPR may underestimate adherence because some patients may have withdrawn from therapy because such therapy is no longer indicated. Migraine frequency and severity fluctuate over time in many patients, and some patients may discontinue prophylaxis because it is no longer warranted. Given the data available, we were unable to assess the ongoing clinical need for prophylaxis and thus may underestimate persistence if a large proportion of patients withdraw from therapy because it is no longer indicated. Despite these limitations, we believe that this study helps provide a broad overview of adherence and persistence to oral migraine prophylaxis and highlight the need for future studies to investigate this topic.

\section{Conclusion}

Observational studies as well as pooled data from RCTs support that oral migraine prophylactics have poor adherence and persistence. New options with improved tolerability and/ or fewer dosing intervals may improve patient adherence to oral migraine prophylaxis treatments. Adherence should be considered as an endpoint in future studies exploring the use of such therapies.

\section{Authors}

ZSOLT HEPP, PharmD, MS, is a Principal Research Associate, Global Health Outcomes Strategy and Research; LISA M. BLOUDEK, PharmD, MS, is Manager, Global Health Outcomes Strategy and Research; and SEPIDEH F. VARON, PhD, is Director, Allergan, Irvine, California.

AUTHOR CORRESPONDENCE: Zsolt Hepp, PharmD, MS, Principal Research Associate, Global Health Outcomes Strategy and Research, Allergan, 2525 Dupont Dr., MI3-110A, Irvine, CA 92612. E-mail: Hepp_Zsolt@Allergan.com. 


\section{DISCLOSURES}

This study was funded by Allergan, Inc., Irvine, California. The sponsor and corresponding authors directed the research design and reviewed all major research decisions (i.e., study instruments, sampling, and analyses). The authors had full access to all data and had final responsibility for the decision to submit for publication. Hepp is a University of Washington/Allergan Pharmaceutical Outcomes Research and Policy Program Fellow, and he is supported by a training grant from Allergan. Bloudek and Varon are employees of Allergan, Inc.

Hepp participated in planning the study, collecting the data, conducting the analysis, and interpreting the results. Bloudek participated in planning the study and interpreting the results. Varon participated in planning the study, collecting the data, conducting the analyses, and interpreting the results. Hepp and Bloudek wrote the first draft. All authors critically reviewed and edited the first draft and subsequent revisions of the manuscript. All authors approved the final manuscript before submission.

The study sponsor funded the study and was involved in the study design, data collection, data analysis, data interpretation, and the writing of the manuscript. Hepp, Bloudek, and Varon were involved in development of study methodology and drafting the manuscript. Hepp had final responsibility for submission of this manuscript.

\section{ACKNOWLEDGMENTS}

The authors wish to acknowledge Imprint Publication Science, New York, New York, for editorial support in the formatting and styling of this manuscript.

\section{REFERENCES}

1. Lipton RB, Bigal ME, Diamond M, et al. Migraine prevalence, disease burden, and the need for preventive therapy. Neurology. 2007;68(5):343-49.

2. Hazard E, Munakata J, Bigal ME, Rupnow MF, Lipton RB. The burden of migraine in the United States: current and emerging perspectives on disease management and economic analysis. Value Health. 2009;12(1):55-64.

3. Ramadan NM, Silberstein SD, Freitag FG, Gilbert TT, Frishberg BM. Evidence-based guidelines for migraine headache in the primary care setting: pharmacological management for prevention of migraine. US Headache Consortium. Available at: http://tools.aan.com/professionals/practice/pdfs/ gl0090.pdf. Accessed November 18, 2013.

4. Kowacs PA, Piovesan EJ, Tepper SJ. Rejection and acceptance of possible side effects of migraine prophylactic drugs. Headache. 2009;49(7):1022-27.

5. Osterberg L, Blaschke T. Adherence to medication. N Engl J Med. 2005;353(5):487-97.

6. Haynes RB, Ackloo E, Sahota N, McDonald HP, Yao X. Interventions for enhancing medication adherence. Cochrane Database Syst Rev. 2008;2:CD000011.

7. Yeaw J, Benner JS, Walt JG, Sian S, Smith DB. Comparing adherence and persistence across 6 chronic medication classes. J Manag Care Pharm. 2009;15(9):728-40. Available at: http://amcp.org/WorkArea/DownloadAsset. aspx?id=8250.

8. Thier SL, Yu-Isenberg KS, Leas BF, et al. In chronic disease, nationwide data show poor adherence by patients to medication and by physicians to guidelines. Manag Care. 2008;17(2):48-52, 55-57.

9. DiMatteo MR, Giordani PJ, Lepper HS, Croghan TW. Patient adherence and medical treatment outcomes: a meta-analysis. Med Care. 2002;40(9):794-811.

10. DiMatteo MR. Variations in patients' adherence to medical recommendations: a quantitative review of 50 years of research. Med Care. 2004:42(3):200-09.

11. Cramer JA, Roy A, Burrell A, et al. Medication compliance and persistence: terminology and definitions. Value Health. 2008;11(1):44-47.

12. Mulleners WM, Whitmarsh TE, Steiner TJ. Noncompliance may render migraine prophylaxis useless, but once-daily regimens are better. Cephalalgia. 1998;18(1):52-56.
13. Peterson AM, Nau DP, Cramer JA, Benner J, Gwadry-Sridhar F, Nichol $\mathrm{M}$. A checklist for medication compliance and persistence studies using retrospective databases. Value Health. 2007;10(1):3-12

14. Garber MC, Nau DP, Erickson SR, Aikens JE, Lawrence JB. The concordance of self-report with other measures of medication adherence: a summary of the literature. Med Care. 2004:42(7):649-52.

15. Nichol MB, Venturini F, Sung JC. A critical evaluation of the methodology of the literature on medication compliance. Ann Pharmacother. 1999;33 (5):531-40

16. Gaul C, van Doorn C, Webering N, et al. Clinical outcome of a headache-specific multidisciplinary treatment program and adherence to treatment recommendations in a tertiary headache center: an observational study. J Headache Pain. 2011;12(4):475-83.

17. Heckman BD, Ellis G. Preventive medication adherence in African American and Caucasian headache patients. Headache. 2011;51(4):520-32.

18. Rothrock JF, Parada VA, Sims C, Key K, Walters NS, Zweifler RM. The impact of intensive patient education on clinical outcome in a clinic-based migraine population. Headache. 2006;46(5):726-31

19. Lafata JE, Tunceli O, Cerghet M, Sharma KP, Lipton RB. The use of migraine preventive medications among patients with and without migraine headaches. Cephalalgia. 2010;30(1):97-104.

20. Berger A, Varon SF, Bramley TJ, et al. Adherence with pharmacological prophylaxis of migraine. Cephalalgia. 2009;29(Suppl 1):S56.

21. Nelles G, Schmitt L, Humbert T et al. Prevention of episodic migraines with topiramate: results from a non-interventional study in a general practice setting. J Headache Pain. 2010;11(1):33-44.

22. Malessa R, Gendolla A, Steinberg B, et al. Prevention of episodic migraine with topiramate: a prospective 24 -week, open-label, flexible-dose clinical trial with optional 24 weeks follow-up in a community setting. Curr Med Res Opin. 2010;26(5):1119-29.

23. Mei D, Capuano A, Vollono C, et al. Topiramate in migraine prophylaxis: a randomised double-blind versus placebo study. Neurol Sci. 2004;25(5):245-50.

24. Lampl C, Huber G, Adl J, et al. Two different doses of amitriptyline ER in the prophylaxis of migraine: long-term results and predictive factors. Eur J Neurol. 2009;16(8):943-48.

25. Krymchantowski AV, Jevoux CC. Topiramate vs divalproex sodium in the preventive treatment of migraine: a prospective "real-world" study. Headache. 2011;51(4):554-58

26. Verspeelt J, De Locht P, Amery WK. Post-marketing cohort study comparing the safety and efficacy of flunarizine and propranolol in the prophylaxis of migraine. Cephalalgia. 1996;16(5):328-36, discussion 288.

27. Rahimtoola H, Buurma H, Tijssen CC, Leufkens HG, Egberts AC. Migraine prophylactic medication usage patterns in The Netherlands. Cephalalgia. 2003;23(4):293-301.

28. Yaldo AZ, Wertz DA, Rupnow MF, Quimbo RM. Persistence with migraine prophylactic treatment and acute migraine medication utilization in the managed care setting. Clin Ther. 2008;30(12):2452-60.

29. Zeller A, Ramseier E, Teagtmeyer A, Battegay E. Patients' self-reported adherence to cardiovascular medication using electronic monitors as comparators. Hypertens Res. 2008;31(11):2037-43.

30. Grymonpre RE, Didur CD, Montgomery PR, Sitar DS. Pill count, selfreport, and pharmacy claims data to measure medication adherence in the elderly. Ann Pharmacother. 1998;32(7-8):749-54.

31. Mohammadianinejad SE, Abbasi V, Sajedi SA. Zonisamide versus topiramate in migraine prophylaxis: a double-blind randomized clinical trial. Clin Neuropharmacol. 2011;34(4):174-77.

32. Cady RK, Schreiber CP, Porter JA, Blumenfeld AM, Farmer KU. A multicenter double-blind pilot comparison of onabotulinumtoxinA and topiramate for the prophylactic treatment of chronic migraine. Headache. 2011;51(1):21-32 
33. Couch JR; Amitriptyline Versus Placebo Study Group. Amitriptyline in the prophylactic treatment of migraine and chronic daily headache. Headache. 2011;51(1):33-51.

34. Rodríguez-Leyva I, Sánchez-Aguilar MC, Hernández-Sierra JF et al. Topiramate vs. amitriptyline in prophylactic treatment of migraine: a controlled clinical trial. Revista Mexicana de Neurociencia. 2010;11(5):338-42.

35. Domingues RB, Silva AL, Domingues SA, Aquino CC, Kuster GW. A double-blind randomized controlled trial of low doses of propranolol, nortriptyline, and the combination of propranolol and nortriptyline for the preventive treatment of migraine. Arq Neuropsiquiatr. 2009;67(4):973-77.

36. Dodick DW, Freitag F, Banks J, et al. Topiramate versus amitriptyline in migraine prevention: a 26-week, multicenter, randomized, double-blind, double-dummy, parallel-group noninferiority trial in adult migraineurs. Clin Ther. 2009;31(3):542-59.

37. Adelman J, Freitag FG, Lainez M, et al. Analysis of safety and tolerability data obtained from over 1,500 patients receiving topiramate for migraine prevention in controlled trials. Pain Med. 2008;9(2):175-85.

38. Ashtari F, Shaygannejad V, Akbari M. A double-blind, randomized trial of low-dose topiramate vs propranolol in migraine prophylaxis. Acta Neurol Scand. 2008;118(5):301-5.

39. Keskinbora K, Aydinli I. A double-blind randomized controlled trial of topiramate and amitriptyline either alone or in combination for the prevention of migraine. Clin Neurol Neurosurg. 2008;110(10):979-84.

40. Krymchantowski AV, Silva MT, Barbosa JS, Alves LA. Amitriptyline versus amitriptyline combined with fluoxetine in the preventative treatment of transformed migraine: a double-blind study. Headache. 2002;42(6):510-14.
41. Diener HC, Matias-Guiu J, Hartung E, et al. Efficacy and tolerability in migraine prophylaxis of flunarizine in reduced doses: a comparison with propranolol 160 mg daily. Cephalalgia. 2002;22(3):209-21.

42. Rao BS, Das DG, Taraknath VR, Sarma Y. A double blind controlled study of propranolol and cyproheptadine in migraine prophylaxis. Neurol India. 2000;48(3):223-26.

43. Kaniecki RG. A comparison of divalproex with propranolol and placebo for the prophylaxis of migraine without aura. Arch Neurol. 1997;54(9):1141-45.

44. Bánk J. A comparative study of amitriptyline and fluvoxamine in migraine prophylaxis. Headache. 1994;34(8):476-78.

45. Pradalier A, Serratrice G, Collard M, et al. Long-acting propranolol in migraine prophylaxis: results of a double-blind, placebo-controlled study. Cephalalgia. 1989;9(4):247-53.

46. Mikkelsen B, Pedersen KK, Christiansen LV. Prophylactic treatment of migraine with tolfenamic acid, propranolol and placebo. Acta Neurol Scand. 1986;73(4):423-27.

47. Ryan RE Sr. Comparative study of nadolol and propranolol in prophylactic treatment of migraine. Am Heart J. 1984;108(4 Pt 2):1156-59.

48. Tfelt-Hansen P, Standnes B, Kangasneimi P, Hakkarainen H, Olesen J. Timolol vs propranolol vs placebo in common migraine prophylaxis: a double-blind multicenter trial. Acta Neurol Scand. 1984;69(1):1-8.

49. Andersson PG, Petersen EN. Propranolol and femoxetine, a HT-uptake inhibitor, in migraine prophylaxis. A double-blind crossover study. Acta Neurol Scand. 1981;64(4):280-88. 